\title{
Time to Remission for Depression with Collaborative Care Management (CCM) in Primary Care
}

Gregory M. Garrison, MD, MS, Kurt B. Angstman, MD, MS, Stephen S. O'Connor, PhD, Mark D. Williams, MD, and Timothy W. Lineberry, MD

Background: Collaborative care management (CCM) has been shown to have superior outcomes to usual care (UC) for depressed patients with a fixed end point. This study was a survival analysis over time comparing CCM with UC using remission (9-item Patient Health Questionnaire [PHQ-9] score <5) and persistent depressive symptoms (PDSs; PHQ-9 score $\geq 10$ ) as end points.

Methods: A retrospective cohort study of 7340 patients with depression cared for at 4 outpatient primary care clinics was conducted from March 2008 through June 2013. All adult patients diagnosed with depression (International Classification of Diseases, 9th Revision [ICD-9], codes 296.2-3) or dysthymia (ICD-9 code 300.4) with an initial PHQ-9 score $\geq 10$ were included. CCM was implemented at all clinics between 2008 and 2010. Kaplan-Meyer survival curves for time to remission and PDSs were plotted. A Cox proportional hazards model was used to adjust for expected differences between patients choosing CCM versus UC.

Results: Median time to remission was 86 days (95\% confidence interval [CI], 81-91 days) for the CCM group versus 614 days (95\% CI, 565-692 days) for the UC group. Likewise, median duration of PDSs was 31 days (95\% CI, 30-33 days) for the CCM group versus 154 days (95\% CI, 138-182 days) for the UC group. In the Cox proportional hazards model, which controlled for covariates such as age, sex, race, diagnosis, and initial PHQ-9 score, CCM was associated with faster remission (hazard ratio of the CCM group [HR $\left.\left.{ }_{\mathrm{CCM}}\right], 2.48 ; 95 \% \mathrm{CI}, 2.31-2.65\right)$.

Conclusions: This study demonstrated that patients enrolled in CCM have a faster rate of remission and a shorter duration of PDSs than patients choosing UC. (J Am Board Fam Med 2016;29:10-17.)

Keywords: Depression, Medical Home, Patient-centered Outcomes Research

Depression is now the second leading cause of disability and poor quality of life in the world. ${ }^{1}$ Depressive symptoms occur in up to $20 \%$ of primary care patients. ${ }^{2}$ Depression leads to dis-

This article was externally peer reviewed.

Submitted 15 April 2015; revised 9 September 2015; accepted 11 September 2015.

From the Department of Family Medicine (GMG, KBA) and the Department of Psychiatry \& Psychology (MDW), Mayo Clinic, Rochester, MN; and the Department of Psychological Sciences, Western Kentucky University, Bowling Green, KY (SSO); Aurora Health Care, Green Bay, WI (TWL).

Funding: Support for this study was provided by the Department of Family Medicine, Mayo Clinic, Rochester, MN.

Conflict of interest: MDW consulted for ACUPERA, a care management company, on algorithms for depression and anxiety, receiving an honorarium only.

Corresponding author: Dr. Gregory M. Garrison, MD, MS, 200 First Street SW, Rochester, MN 55905 (E-mail: garrison. gregory@mayo.edu). rupted interpersonal relationships, substance abuse, lost work time, and suicide. ${ }^{3}$ Quality of life in depressed patients is inversely related to the severity of depressive symptoms but can be improved with treatment. ${ }^{3-5}$ Thus, depression can be made less burdensome by speeding up the time to remission of depressive symptoms using better treatments.

Treatment for patients with major depressive disorder has recently centered around a collaborative care management (CCM) model. ${ }^{6-8}$ Compared with usual care (UC), CCM has demonstrated greater likelihood of clinical remission in primary care practices, decreased evidence of persistent depressive symptoms, decreased likelihood of short-term recurrence, and improved workplace outcomes. $^{9-14}$ Several studies using the 9-item $\mathrm{Pa}$ tient Health Questionnaire (PHQ-9) demonstrated 
responses to CCM occur as early as 6 to 12 weeks. ${ }^{15-17}$ Retrospective studies of data collected in our clinical registry using logistic regression techniques have demonstrated the effectiveness of CCM over UC in terms of remission, persistent depressive symptoms (PDSs), short-term recurrence, and prolonged treatment at 6 - and 12month fixed intervals. ${ }^{18,19}$

However, the above-referenced studies evaluated depression at baseline and then at fixed end points. This type of analysis is akin to determining how many runners cross the finish line of a marathon by 5 hours. While they are useful data about the group's overall performance, what matters to each individual runner is how quickly he or she finish the race. Similarly, for an individual patient struggling with work and relationships because of major depression, the outcome that matters to them is how quickly they will recover and whether CCM will speed up their recovery. Because survival analysis utilizes time data, it is better suited to answering this question in the setting of chronic diseases (such as major depression) than logistic regression techniques. ${ }^{20}$

Relatively few publications have used survival analysis techniques to assess and compare clinical improvement following a diagnosis of major depression or dysthymia. McLeod et $\mathrm{al}^{21}$ used survival analysis to determine the speed of recovery from major depression in a small sample of married patients. Unutzer et $\mathrm{al}^{22}$ plotted Kaplan-Meyer curves comparing the time to improvement of depressive symptoms before and after implementation of a pay-for-performance incentive program. Another study used a Cox proportional hazards model to assess whether substance abuse treatment referral improved the response to depression treatment. ${ }^{23}$ Vemer et $\mathrm{al}^{24}$ created a survival model to predict return to work in patients with major depression.

In this study we use survival analysis to compare the rate at which patients improve when treated for depression by either CCM or UC, thus providing more information than previous studies with fixed follow-up intervals about the performance of CCM compared with UC. In addition, survival analysis makes use of all the available data, even for patients who are lost to follow-up or fail to complete treatment. ${ }^{25}$ The hypothesis is that, after controlling for baseline demographic characteristics and depres- sion severity, enrollment in CCM results in a faster rate of remission and shorter duration of PDSs when compared with UC.

\section{Methods}

\section{Environment}

CCM for depressed patients began in March 2008 at the primary care sites within the Mayo Clinic, Rochester, Minnesota. By March 2010, CCM was available to all 100,000 adult patients cared for within our Department of Family Medicine, Division of Primary Care Internal Medicine, and Division of Community Pediatrics and Adolescent Medicine at 4 outpatient clinical sites - all of which are certified as patient-centered medical homes (PCMHs). CCM helped the primary care providers (PCPs) manage the care of their depressed patients via assistance from a specially trained nurse care manager and consultations with a psychiatrist. Any adult patient diagnosed by their primary care provider with major depression (International Classification of Diseases, 9th Revision [ICD-9] codes 296.2 to 296.3) or dysthymia (ICD-9 code 300.4) and an initial PHQ-9 score of $\geq 10$ has the option of enrolling in the program. Only patients formally diagnosed with bipolar disorder (ICD-9 codes 296.4 to 296.8 ) were excluded from the program. The CCM processes have been previously been reviewed. ${ }^{18,19}$

Briefly, the components of the CCM model in clinical practice include an electronic depression registry, specially trained registered nurses as care managers, use of clinical guidelines for care management, and an integrated behavioral health team consisting of psychologists, social workers, and clinical nurse specialists with a supervising psychiatrist. Decisions regarding treatment with prescription medications, outside referrals for counseling, and physician follow-up visits remained with the PCP. The integrated behavioral health team supported the PCP with case review, treatment recommendations, and brief counseling, while the care managers provided ongoing contact with the patients via telephone calls and office visits.

PCPs were encouraged to discuss the CCM program with their eligible patients at the time of initial diagnosis. The care managers were available to do an in-person "warm hand-off," during which they would explain the program and its components to patients both verbally and with printed literature. There was no additional cost to patients 


\begin{tabular}{|c|c|c|c|c|}
\hline & All $(\mathrm{n}=7349)$ & $\mathrm{CCM}(\mathrm{n}=3595)$ & $\mathrm{UC}(\mathrm{n}=3754)$ & $P$ Value* \\
\hline Age (years), mean (SD) & $41.8(16.5)$ & $41.1(15.7)$ & $42.5(17.2)$ & .0080 \\
\hline Female sex, n (\%) & $5169(70.4)$ & $2620(73.1)$ & $2549(67.9)$ & $<.0001$ \\
\hline Marital status (married) , n (\%) & $3767(51.3)$ & $1890(52.7)$ & $1877(50.0)$ & .023 \\
\hline Race (white), n (\%) & $6721(91.6)$ & $3347(93.3)$ & $3374(89.9)$ & $<.0001$ \\
\hline Clinical site, n (\%) & & & & $<.0001$ \\
\hline A & $3547(48.3)$ & $1164(32.5)$ & $2383(63.5)$ & \\
\hline $\mathrm{B}$ & $950(12.9)$ & $464(12.9)$ & $486(13.0)$ & \\
\hline $\mathrm{C}$ & $1503(20.5)$ & $954(26.5)$ & $549(14.6)$ & \\
\hline $\mathrm{D}$ & $1340(18.3)$ & $1004(28.0)$ & $336(9.0)$ & \\
\hline Diagnosis code, n (\%) & & & & $<.0001$ \\
\hline $296.2 x$ & $3729(50.8)$ & $1866(52.0)$ & $1863(49.6)$ & \\
\hline $296.3 x$ & $2596(35.4)$ & $1427(39.8)$ & $1169(31.1)$ & \\
\hline 300.4 & $1015(13.8)$ & $293(8.2)$ & $722(19.2)$ & \\
\hline Initial PHQ-9 score, mean (SD) & $15.4(4.1)$ & $15.6(4.1)$ & $15.1(4.1)$ & $<.0001$ \\
\hline Moderate, n (\%) & 3564 (48.6) & $1633(45.5)$ & $1931(51.4)$ & \\
\hline Moderately severe, n (\%) & $2485(33.9)$ & $1287(35.9)$ & $1198(31.9)$ & \\
\hline Severe, n (\%) & $1291(17.6)$ & $666(18.6)$ & $625(16.6)$ & \\
\hline
\end{tabular}

${ }^{*}$ Calculated using the $\chi^{2}$ test for categorical variables and Wilcoxon rank sum test for ordinal variables.

CCM, collaborative care management; PHQ-9, 9-item Patient Health Questionnaire; SD, standard deviation; UC, usual care.

to participate in CCM. Patients were encouraged to review the literature and were free to enroll, decline, or defer decision making to a follow-up telephone call.

As of June 30, 2013, our depression registry had records of 7340 unique primary care patients with at least 1 episode of depression and $>50,000$ separate PHQ-9 scores. Patients who may have had a relapse during the study time frame only had data from the initial episode included.

\section{Study Design}

This study is a retrospective cohort study regarding a CCM process started in March 2008. We utilized an existing registry to identify depressed patients who were eligible for CCM from March 2008 through June 2013 at clinical sites that had fully implemented the CCM process. Only patients with a signed authorization allowing review of their medical records for research purposes were included in the study. The study was reviewed and approved by our institutional review board.

Baseline data including CCM enrollment status, date of eligibility for CCM, age at eligibility, sex, marital status (married or not), race (white or not), clinical site, initial ICD-9 diagnosis code, and initial PHQ-9 score were recorded. In addition, all follow-up PHQ-9 scores through June 2013 were collected for each subject. The number of additional follow up PHQ-9 scores was variable for each patient and was influenced by initial depression severity, individual treatment plans, provider recommendations, and patient compliance. In general, they were obtained at all office visits and during triage telephone calls for both CCM and UC patients, with additional PHQ-9 assessments performed approximately monthly by the care managers for CCM patients. Under intention-totreat assumptions, those who initially enrolled in CCM remained in that group, even if they later withdrew ( $\mathrm{n}=481 ; 13.5 \%)$. Remission of depressive symptoms was defined as a PHQ-9 score $<5$. Likewise, PDSs were defined as a PHQ-9 score $\geq 10$.

\section{Data Analysis}

All independent variables were summarized by group (CCM vs UC). For categorical data, a $\chi^{2}$ test was used to assess differences between groups; $P<$ .05 considered statistically significant. Likewise, a Wilcoxon rank sum test was used to assess group differences for the numeric variables.

A Kaplan-Meyer survival curve was graphed to illustrate differences in time to remission between those enrolled in CCM and those receiving UC. 
Figure 1. Time to remission (9-item Patient Health Questionnaire score <5) with collaborative care management versus usual care

Time to Remission

(PHQ9 < 5)

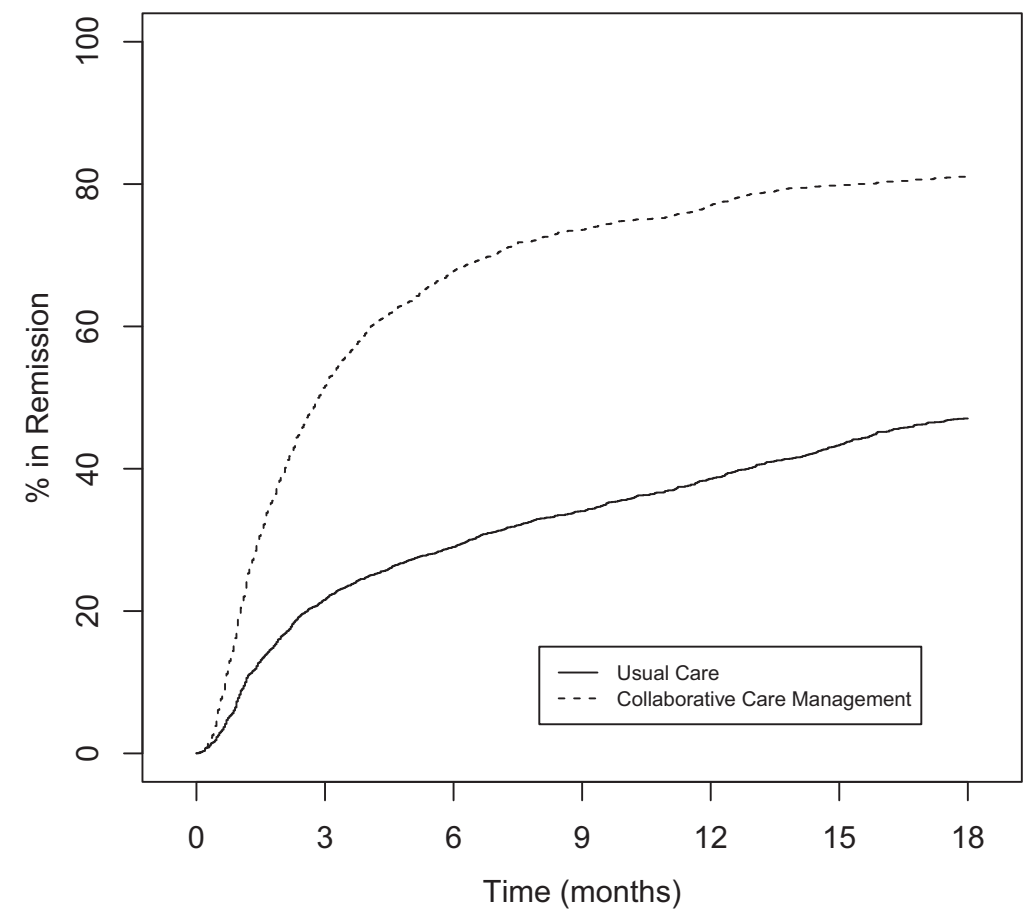

The time to remission was defined as the number of days that elapsed between a given patient's eligibility for CCM (regardless of whether they choose to engage in CCM) and their first subsequent PHQ-9 score $<5$. The median time to remission was calculated with $95 \%$ confidence intervals (CIs). In addition, the null hypothesis that the 2 remission curves were the same was compared statistically using a $\chi^{2}$ test, with $P<.05$ considered significant.

To adjust for expected differences between the subjects choosing CCM versus those choosing UC, a Cox proportional hazards model was also used to analyze the data ( $\mathrm{R}$ version 3.0.2). Covariates, selected based on prior work, ${ }^{10,12,18}$ include age, sex, marital status, race, depression diagnosis, the initial PHQ-9 score, and the treatment variable of enrollment in CCM. Two Cox models were used, 1 with a dependent variable of remission and the other with a dependent variable of PDSs. $P$ values $<.05$ were considered statistically significant, and $95 \%$ CIs were calculated for each hazard ratio (HR). An $\mathrm{HR}>1$ indicates the association of a covariate with faster remission of depressive symptoms.

\section{Results}

In all, 7340 eligible subjects in the registry were analyzed; of these, 3586 (48.9\%) initially enrolled in CCM. There were small but statistically significant differences in the demographics of subjects choosing CCM versus those choosing UC (Table 1). Patients choosing CCM were slightly younger (mean, 41.1 vs 42.5 years; $P=.008$ ) and more likely to be female $(73.1 \%$ vs $67.9 \% ; P<.001)$, married (52.7\% vs $50.0 \% ; P=.022)$, and white $(93.3 \%$ vs $89.9 \% ; P<.001)$. The initial PHQ-9 scores were slightly higher among CCM patients (mean, 15.6 vs 15.1; $P<.001)$, with more PHQ-9 scores obtained (median, 2 vs $5 ; P<.001$ ) and fewer dysthymia diagnoses $(8.2 \%$ vs $19.2 \% ; P<.001)$.

Figure 1 shows the Kaplan-Meier style time-toremission curve for CCM versus UC during the first 18 months of follow-up. There were significant differences in time to remission between the groups $(P<.001)$. The median time to remission in the CCM group was 86 days (95\% CI, 81-91 days) versus 614 days (95\% CI, 565-692 days) in the UC group. Similarly, Figure 2 shows a significant dif- 
Figure 2. Persistent depressive symptoms (9-item Patient Health Questionnaire score $\geq 5$ ) with collaborative care management versus usual care

Persistent Depressive Symptoms

(PHQ9 >= 10)

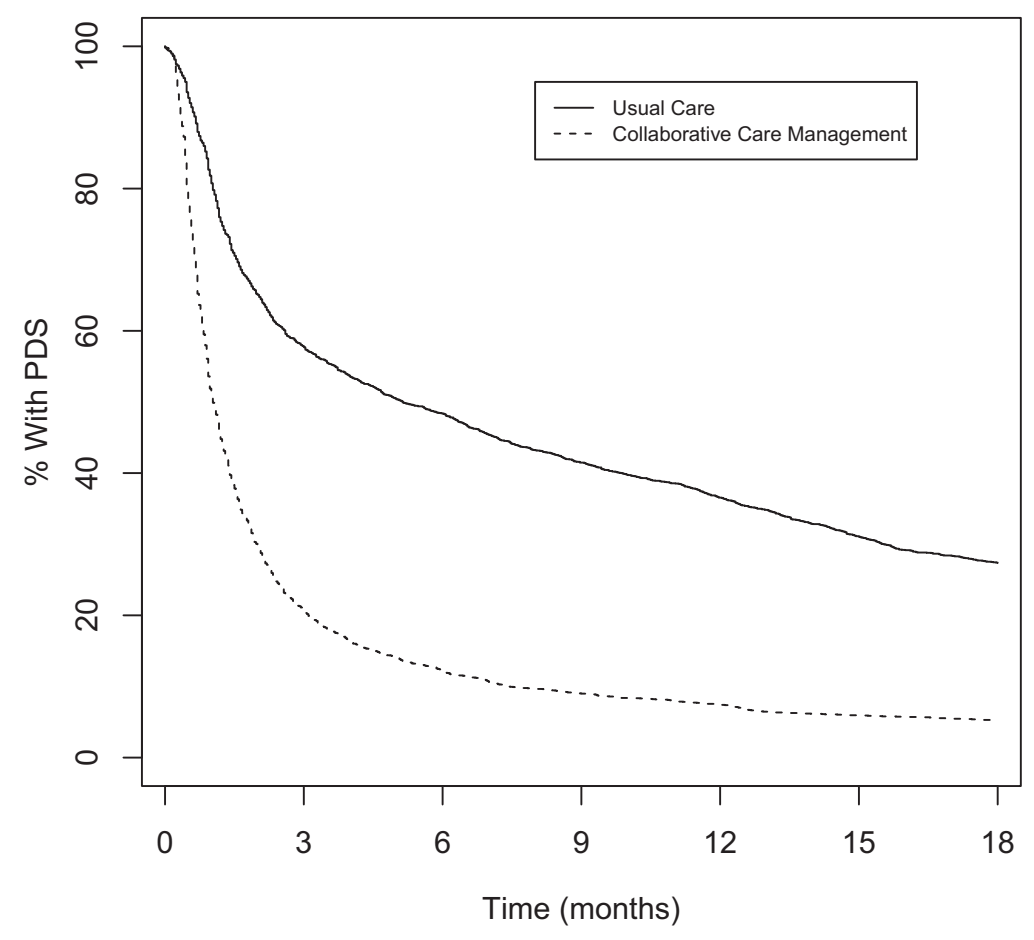

ference in the PDSs between the CCM and UC groups $(P<.001)$. The median duration of PDSs in the CCM group was 31 days (95\% CI, 30-33 days) versus 154 days (95\% CI, 138-182 days) for the UC group.

To further investigate the time to remission and adjust for the known differences between groups, a Cox proportional hazard model was used (Table 2). CCM patients were 2.5 times more likely to experience remission sooner (HR, 2.48; 95\% CI, 2.312.65) when compared with UC patients. Patients $\geq 65$ years old were more likely to experience remission sooner than younger patients (HR of 1.43 among those aged 65 to 79 years and 1.29 among those aged $\geq 80$ years). Likewise, married patients were more likely to experience remission sooner (HR, 1.18) than unmarried patients. Sex and race made no difference. As expected, those with a diagnosis of recurrent depression were less likely to experience remission sooner (HR, 0.87) than those with initial depression, but those with dysthymia showed no difference. Higher initial PHQ-9 scores were less likely to achieve sooner remission (moderately severe: HR, 0.80; severe: HR, 0.71). The clinical site also made a difference: 2 of the 3 satellite clinics were more likely to show remission sooner.

\section{Discussion}

Results from our study demonstrate that, consistent with our hypothesis, patients enrolled in CCM have a faster rate of remission and shorter duration of PDSs than patients treated with UC. In fact, patients enrolled in CCM are 2.5 times more likely to experience remission sooner than patients treated with UC. Their median time to remission was 86 days. Thus, $50 \%$ of patients treated with CCM are expected to achieve remission within 3 months, suggesting that this may be a good time to reevaluate the clinical efficacy of treatment plans among individual patients.

One possible explanation for the differences between CCM and UC might be related to challenges surrounding the escalation of care when the patient is not responding to initial treatment. Compared with $\mathrm{CCM}$, in $\mathrm{UC}$, patients may need to initiate contact with their health care providers to get ad- 
Table 2. Cox Proportional Hazard Model for Remission of Depressive Symptoms

\begin{tabular}{|c|c|c|}
\hline & $\begin{array}{l}\text { Hazard Ratio for Remission* } \\
\qquad(95 \% \mathrm{CI})\end{array}$ & $P$ Value \\
\hline \multicolumn{3}{|l|}{ Group } \\
\hline UC & Reference & \\
\hline CCM & $2.48(2.31-2.65)$ & $<.0001$ \\
\hline \multicolumn{3}{|l|}{ Age (years) } \\
\hline $18-50$ & Reference & \\
\hline $51-64$ & $1.00(0.93-1.08)$ & .979 \\
\hline $65-79$ & $1.43(1.27-1.60)$ & $<.0001$ \\
\hline$\geq 80$ & $1.29(1.06-1.58)$ & .012 \\
\hline Sex & & .81 \\
\hline Female & Reference & \\
\hline Male & $1.01(0.94-1.08)$ & \\
\hline Marital status & & $<.0001$ \\
\hline Not married & Reference & \\
\hline Married & $1.18(1.11-1.26)$ & \\
\hline Race & & .113 \\
\hline White & Reference & \\
\hline Nonwhite & $0.91(0.80-1.02)$ & \\
\hline \multicolumn{3}{|l|}{ Clinic } \\
\hline A & Reference & \\
\hline B & $1.03(0.93-1.14)$ & .563 \\
\hline $\mathrm{C}$ & $1.34(1.24-1.45)$ & $<.0001$ \\
\hline $\mathrm{D}$ & $1.27(1.17-1.38)$ & $<.0001$ \\
\hline \multicolumn{3}{|l|}{ Diagnosis } \\
\hline Initial & Reference & \\
\hline Recurrent & $0.87(0.82-0.93)$ & $<.0001$ \\
\hline Dysthymia & $0.98(0.89-1.08)$ & .681 \\
\hline \multicolumn{3}{|l|}{ Initial PHQ-9 score } \\
\hline Moderate (10-14) & Reference & \\
\hline $\begin{array}{l}\text { Moderately } \\
\text { severe (15-19) }\end{array}$ & $0.80(0.75-0.85)$ & $<.0001$ \\
\hline Severe (20-27) & $0.71(0.65-0.77)$ & $<0.0001$ \\
\hline
\end{tabular}

${ }^{*}$ Remission is defined as a 9-item Patient Health Questionnaire (PHQ-9) score $<5$.

CCM, collaborative care management; CI, confidence interval; PHQ-9, 9-item Patient Health Questionnaire; UC, usual care.

ditional attention. Hesitation by the patient, anhedonia associated with depression, and barriers to accessing care, such as having to take time off work for visits, may delay improvement. With CCM, the concept of rapid escalation of treatment is built into the model via regular scheduled contact with care managers and periodic case review by a supervising psychiatrist. Thus slow responders in CCM are less likely to go unnoticed for long periods of time.

While other studies have confirmed the superiority of CCM at specific follow-up intervals, such as 6 or 12 months, ${ }^{16,18,19}$ our study is unique in showing its effectiveness over time using survival analysis techniques. This methodology has the advantage of using all the available data, even for patients who are lost to follow-up or fail to complete treatment. ${ }^{25}$ Because our time series data are much more granular, we are able to show divergence within the first month of treatment with the Kaplan-Meier curves in Figures 1 and 2.

Patients care about how quickly they recover from depression. The existing logistic regression models studying recovery from depression neglect valuable time-dependent information about the speed of recovery. Because of this shortcoming, logistic regression analysis is dependent on the length of the follow-up period chosen. For chronic diseases such as depression, it only approximates the survival model for short follow-up periods and rare disease incidence. ${ }^{20}$ Therefore, we suggest that future studies of differing CCM models within PCMHs should use survival analysis techniques as an adjunct to other methodologies. Based on our results showing rapid improvement, simply showing superiority to $\mathrm{UC}$ or to an existing CCM at a single follow-up point in time is insufficient information to fully compare care models.

CCM for depression embodies many of the principals advocated for the PCMH, such as physician-directed medical care, care coordination, and enhanced access. ${ }^{26}$ However, as Friedberg et $\mathrm{al}^{27}$ recently point out, the PCMH has failed to deliver on overall utilization or cost reduction, perhaps because it is an "expensive technology" that is not well suited for widespread implementation in a nontargeted manner. ${ }^{28}$ Thus the focus has shifted to applying PCMH innovations to targeted populations. As our study shows, CCM can be applied to a targeted population with good results.

\section{Limitations}

A major limitation of our study is its nonrandomized, retrospective nature. We cannot entirely eliminate bias because of self-selection into CCM. Since CCM has previously been shown to be superior to UC at 6 months, ${ }^{18,19}$ it could be considered unethical to randomize patients to an inferior treatment for a disease that carries significant morbidity and mortality. Thus we were left to analyze the differences between treatments chosen freely by patients and their physicians. We attempted to control for known confounding covariates using our Cox proportional hazard model, but unknown 
confounders could be affecting a patient's choice of CCM versus UC.

In addition, CCM patients had more PHQ-9 scores recorded than UC patients, leading to greater granularity of data. This could give a false impression of faster remission simply because the PHQ-9 was sampled sooner. However, if greater granularity were the only factor, we would expect the Kaplan-Meyer curves to eventually converge. This is clearly not the case over the 18 -month follow-up time frame shown, nor does it happen when we examined longer follow-up time frames. A prospective study using the same regular interval of PHQ-9 screening for both CCM and UC would eliminate this source of bias. However, by establishing the contact necessary to obtain the PHQ-9 scores at regular intervals, such a prospective study would fundamentally alter what is considered UC, making any conclusions suspect. In any case, the number of PHQ-9 scores we collected was similar to the number found in a large comparative effectiveness study conducted by Valuck et al. ${ }^{29}$

Our study was performed at a large multisite primary care practice using a unified CCM model. Further study is necessary to determine whether smaller practice settings and more heterogeneous populations also demonstrate faster resolution of depressive symptoms with CCM. Finally, we selected the PHQ-9 score as our end point; thus this study does not assess other possible patient-oriented outcomes such as quality of life, return to work, healthcare utilization, suicide attempts, or medication usage. Further study is needed to determine whether faster remission via CCM is associated with improved healthcare utilization and overall cost.

The authors thank Isaac Johnson for collecting the data necessary for this study.

\section{References}

1. Ferrari AJ, Charlson FJ, Normal RE, et al. Burden of depressive disorders by country, sex, age, and year: findings from the global burden of disease study 2010. PLoS Med 2013;10:e1001547.

2. Zung WW, Broadhead WE, Roth ME. Prevalence of depressive symptoms in primary care. J Fam Pract 1993;37:337-44.

3. IsHak WW, Greenberg JM, Balayan K, et al. Quality of life: the ultimate outcome measure of inter- ventions in major depressive disorder. Harv Rev Psychiatry 2011;19:229-39.

4. Papakostas GI, Peterson T, Mahal Y, Mischoulon D, Nierenberg AA, Fava M. Quality of life assessments in major depressive disorder: a review of the literature. Gen Hosp Psychiatry 2004;26:13-7.

5. Pyne JM, Patterson TL, Kaplan RM, Gillin JC, Koch WL, Grant I. Assessment of the quality of life of patients with major depression. Psychiatr Serv 1997;48:224-30.

6. Unutzer J, Katon W, Callahan CM, et al. Collaborative care management of late-life depression in the primary care setting: a randomized controlled trial. JAMA 2002;288:2836-45.

7. Thota AB, Sipe TA, Byard GJ; et al. Collaborative care to improve the management of depressive disorders: a community guide systematic review and meta-analysis. Am J Prev Med 2012;42:525-38.

8. Gilbody S, Bower P, Fletcher J, Richards D, Sutton AJ.. Collaborative care for depression: a cumulative meta-analysis and review of longer-term outcomes. Arch Intern Med 2006;166:2314-21.

9. Angstman KB, Dejesus RS, Rohrer JE. Correlation between mental health co-morbidity screening scores and clinical response in collaborative care treatment for depression. Ment Health Fam Med 2010;7:129-33.

10. Angstman KB, MacLaughlin KL, Rasmussen NH, DeJesus RS, Katzelnick DJ. Age of depressed patient does not affect clinical outcome in collaborative care management. Postgrad Med 2011;123:122-8.

11. Angstman KB, Maclaughlin KL, Williams MD, Rasmussen NH, Dejesus RS.. Increased anxiety and length of treatment associated with depressed patients who are readmitted to collaborative care. J Prim Care Community Health 2011;2:82-6.

12. Angstman KB, Pecina JL, Bernard ME, Matthews MR.. Prolonged care management for depression: a case-controlled study of those enrolled for more than one year. $\mathrm{J}$ Prim Care Community Health 2013;4:129-34.

13. Angstman KB, Shippee ND, Maclaughlin KL, et al. Patient self-assessment factors predictive of persistent depressive symptoms 6 months after enrollment in collaborative care management. Depress Anxiety 2013;30:143-8.

14. Wang PS, Simon GE, Avorn J, et al. Telephone screening, outreach, and care management for depressed workers and impact on clinical and work productivity outcomes: a randomized controlled trial. JAMA 2007;298:1401-11.

15. Spitzer RL, Kroenke K, Williams JB. Validation and utility of a self-report version of PRIME-MD: the PHQ primary care study. Primary Care Evaluation of Mental Disorders. Patient Health Questionnaire. JAMA 1999;282:1737-44.

16. Angstman KB, Rohrer JE, Rasmussen NH. PHQ-9 response curve: rate of improvement for depression treatment with collaborative care management. J Prim Care Community Health 2012;3:155-8. 
17. Shippee ND, Shah ND, Angstman KB, et al. Impact of collaborative care for depression on clinical, functional, and work outcomes: a practice-based evaluation. J Ambul Care Manage 2013;36:13-23.

18. Angstman KB, Pietruszewski P, Rasmussen NH, Wilkinson JM, Katzelnick DJ. Depression remission after six months of collaborative care management: role of initial severity of depression in outcome. Ment Health Fam Med 2012;9:99-106.

19. Williams M, Angstman K, Johnson I, Katzelnick D. Implementation of a care management model for depression at two primary care clinics. J Ambul Care Manage 2011;34:163-73.

20. Green MS, Symons MJ A comparison of the logistic risk function and the proportional hazards model in prospective epidemiologic studies. J Chronic Dis 1983;36:715-23.

21. McLeod JD, Kessler RC, Landis KR. Speed of recovery from major depressive episodes in a community sample of married men and women. J Abnorm Psychol 1992;101:277-86.

22. Unützer J, Chan YF, Hafer E, et al. Quality improvement with pay-for-performance incentives in integrated behavioral health care. Am J Public Health 2012;102:e41-5.

23. Chan YF, Huang H, Bradley K, Unützer J. Referral for substance abuse treatment and depression improvement among patients with co-occurring disor- ders seeking behavioral health services in primary care. J Subst Abuse Treat 2014;46:106-12.

24. Vemer P, Bouwmans CA, Zijlstra-Vlasveld MC, van der Feltz-Cornelis CM, Hakkaart-van Roijen L. Let's get back to work: survival analysis on the return-to-work after depression. Neuropsychiatr Dis Treat 2013;9:1637-45.

25. Spruance SL, Reid JE, Grace M, Samore M. Hazard ratio in clinical trials. Antimicrob Agents Chemother 2004;48:2787-92.

26. Patient-Centered Primary Care Collaborative. Joint principles of the patient-centered medical home. February 2007. Available from: http://www.aafp.org/dam/AAFP/ documents/practice_management/pcmh/initiatives/ PCMHJoint.pdf. Accessed November 13, 2015.

27. Friedberg MW, Schneider EC, Rosenthal MB, Volpp KG, Werner RM. Association between participation in a multipayer medical home intervention and changes in quality, utilization, and costs of care. JAMA 2014;311:815-25.

28. Schwenk TL. The patient-centered medical home: one size does not fit all. JAMA 2014;311:802-3.

29. Valuck RJ, Anderson HO, Libby AM, et al. Enhancing electronic health record measurement of depression severity and suicide ideation: a Distributed Ambulatory Research in Therapeutics Network (DARTNet) study. J Am Board Fam Med 2012;25: 582-93. 